\title{
6 Analyse: Quantitäten
}

Zusammenfassung: Im Kapitel werden quantitative Befunde dargelegt, die sich auf der Grundlage der erfolgten Annotationen der über 520 SN-Token ergeben. Das erhobene Inventar wird zunächst auf die Inventarbildung selbst, also hinsichtlich dessen Verteilung auf die jeweiligen Orte bzw. Ortstypen und auf die jeweiligen Benennungszeiträume, dargelegt. Die Konstruktionen zeichnen durch ein außerordentlich hohes Maß an Systematizität aus: Über $440 \mathrm{SN}$ Token können 27 MOD-Types zugeschrieben werden, die im kolonialen Namenprojekt der deutschen Metropole ortsübergreifende Verwendung fanden. In diachroner Perspektive lassen sich Korrelationen zwischen Namenmustern und gesellschaftspolitischen Benennungsphasen feststellen. Davon können nichtusuelle einzelortsbezogene Phänomene, die sich zahlenmäßig auf die annektierte Stadt Lodz bzw. Litzmannstadt [Łódź] verdichten, abgegrenzt werden.

\subsection{Ortstypen}

In Kap. 4 wurde bereits dargelegt, dass sich kolonial motivierte Straßenvergabepraktiken nicht nur auf die administrativen und wirtschaftlichen Kolonialmetropolen beschränkten: Das erhobene Gesamtinventar an über 520 (527) SNToken wurde nachweislich in den öffentlichen Raum von nahezu 100 (99) Städten des nach historischen Einwohnerzahlen angelegten Ortskorpus des Deutschen Reichs verfügt. In folgender Liste werden die für den Untersuchungszeitraum relevanten historischen Städte angegeben. Alle Groß- und Mittelstädte der nach 1945 abgetrennten Gebiete wurden mit den heutigen nicht-deutschsprachigen Ortsbezeichnungen in eckigen Klammern versehen:

Altena, Altenburg, Amberg, Asch [Aš], Bad Godesberg, Bautzen, Berlin, Bochum, Bottrop, Brandenburg an der Havel, Braunschweig, Bremen, Breslau [Wrocław], Chemnitz, Cuxhaven, Danzig [Gdańsk], Delmenhorst, Dessau, Dresden, Duisburg, Düsseldorf, Eilenburg, Erfurt, Essen, Forst (Lausitz), Frankfurt am Main, Frankfurt (Oder), Gelsenkirchen, Glatz [Kłodzko], Gleiwitz [Gliwice], Gmünd bzw. Schwäbisch Gmünd, Gotha, Hagen, Hamburg, Hannover, Heilbronn, Herford, Hildesheim, Hindenburg O.S. [Zabrze], Iglau [Jihlava], Iserlohn, Kaiserslautern, Karlsbad [Karlovy Vary], Karlsruhe, Kassel, Kattowitz [Katowice], Kiel, Koblenz, Köln, Königsberg i. Pr. bzw. Königsberg (Pr.) [Kaliningrad], Königshütte [Chorzów], Landsberg an der Warthe [Gorzów Wielkopolski], Leipzig, Leslau [Włocławek], Lodz bzw. Litzmannstadt [€ódź], Lübeck, Ludwigsburg, Ludwigshafen am Rhein, Lüneburg, Lünen, Magdeburg, Mannheim, Merseburg, Mühlheim an der Ruhr, München, München Gladbach, Münster, Neustadt an der Haardt bzw. Weinstraße, Nürnberg, Oberhausen, Oldenburg, Oranienburg, Pabianitz [Pabianice], Pirmasens, Pirna, Posen [Poznań], Ravensburg, Recklinghausen, Riesa, Saalfeld, Saarbrücken, Saarlouis bzw. Saarlautern, Schweidnitz [Świdnica], 
Schweinfurt, Siegburg, Solingen, Stettin [Szczecin], Stolberg (Rhld.), Stuttgart, Swinemünde [Świnoujście], Tübingen, Völklingen, Waldenburg [Wałbrzych], Weimar, Wiesbaden, Wolfenbüttel, Wuppertal, Zerbst/Anhalt, Zwickau.

Die vorgenommenen Annotationen jedes SN-Tokens umfassten dabei auch den jeweiligen Ortstyp: Die Städte wurden mit Informationen nach ihrer historischen Größe versehen oder es erfolgten Zuordnungen, die deren Eingliederung in nationalsozialistischer Zeit unmittelbar vor (Münchener Abkommen, Protektorat Böhmen und Mähren) bzw. mit (Polenfeldzug) Kriegsbeginn aufzeigen. Die Kategorisierung des Gesamtinventars kolonial motivierter Straßenbenennungen hinsichtlich der Ortstypen führte zu folgender Verteilung:

Tab. 5: Verteilung der SN-Token auf die jeweiligen Ortstypen.

\begin{tabular}{|c|c|c|c|}
\hline & Großstädte & Mittelstädte & Städte der annektierten Gebiete \\
\hline Anzahl an Orten & 45 & 44 & 10 \\
\hline Anzahl an SN-Token & 336 & 136 & 55 \\
\hline $\begin{array}{l}\text { Prozentuale Aufteilung der SN- } \\
\text { Token auf die jeweiligen Ortstypen }\end{array}$ & ca. $64 \%$ & ca. $26 \%$ & ca. $10 \%$ \\
\hline
\end{tabular}

Die Anzahl der jeweiligen Einschreibungsorte an Groß- und Mittelstädten hält sich dabei relativ die Waage: Bei kolonialen Straßenbenennungen im öffentlichen Raum des Deutschen Reichs handelt es sich somit um ortsübergreifendnationale Praktiken, die in Groß- wie Mittelstädten und darüber hinaus für singuläre Städte der in nationalsozialistischer Zeit annektierten Gebiete am Ende der 1930er Jahre erhoben werden konnten. Trotz der nahezu übereinstimmenden Anzahl von Groß- und Mittelstädten, für die koloniale Namenvergabepraktiken bis 1945 nachzuweisen sind, ist dennoch festzustellen, dass sich koloniale Namenvergabepraktiken in quantitativer Hinsicht vor allem auf die Großstädte konzentrierten. Das betrifft die hohe Verteilungsrate für Großstädte: Für über die Hälfte der 70 Großstädte, für die zwischen 1884 und 1945 über 100.000 Einwohner zu konstatieren sind, konnten nachweislich kolonial motivierte Namenvergabepraktiken erhoben werden. ${ }^{111}$ Die Zahl an Mittelstädten, die hin-

111 D. s.: Berlin, Bochum, Braunschweig, Bremen, Breslau [Wrocław], Chemnitz, Dessau, Dresden, Duisburg, Düsseldorf, Erfurt, Essen, Frankfurt am Main, Gelsenkirchen, Glatz [Kłodzko], Gleiwitz [Gliwice], Hagen, Hamburg, Hannover, Hindenburg O.S. [Zabrze], Kaiserslautern, Karlsruhe, Kassel, Kiel, Köln, Königsberg i. Pr. bzw. Königsberg (Pr.) [Kaliningrad], Leipzig, Lübeck, Ludwigshafen am Rhein, Magdeburg, Mannheim, Mühlheim an der Ruhr, München, Mün- 
sichtlich etwaiger kolonial motivierter Benennungen untersucht wurden, fällt auch nach Abzug der mehrfachen Zuschnitte mit über 300 entsprechenden Orten viel größer aus. Nichtsdestotrotz konnten nur für 44 Mittelstädte koloniale Benennungen bis 1945 erhoben werden (etwa 16\%). Die vorherige Tabelle verdeutlicht darüber hinaus auch, dass die Anzahl an kolonial motivierten Straßennamenverfügungen in den Großstädten des Deutschen Reichs zahlenmäßig weitaus höher ausfällt: Im Durchschnitt wurden in den relevanten Großstädten etwa sieben kolonial motivierte SN-Token verfügt, während sich die Zahl in den Mittelstädten auf etwa drei SN-Token beläuft.

Über ein Zehntel des erstellten Inventars konnte für zehn Städte derjenigen Gebiete erhoben werden, die erst Ende der 1930er Jahre vom nationalsozialistischen Deutschen Reich im Zuge der Besetzung bzw. durch den direkten Angriff annektiert wurden.

Asch [Aš], Danzig [Gdańsk], Iglau [Jihlava], Karlsbad [Karlovy Vary], Kattowitz [Katowice], Königshütte [Chorzów], Leslau [Włocławek], Lodz bzw. Litzmannstadt [Łódź], Pabianitz [Pabianice], Posen [Poznań].

Die Anzahl der erhobenen SN-Token fällt dabei sehr unterschiedlich aus: Für die Orte Asch [Aš], Iglau [Jihlava], Karlsbad [Karlovy Vary], Kattowitz [Katowice] und Leslau [Włocławek] konnten nur singuläre kolonial motivierte Namen erhoben werden. Für Danzig [Gdańsk], Königshütte [Chorzów], Pabianitz [Pabianice] und Posen [Poznań] wurden zwischen vier bis acht kolonial motivierte Benennungen identifiziert und inventarisiert. Die in Lodz bzw. Litzmannstadt [Łódź] erhobenen 29 SN-Token, die als Cluster in Stadtplänen und/oder Adressbüchern für den Beginn der 1940er Jahre erhoben werden konnten, stellen sowohl in quantitativer Hinsicht als auch in Bezug auf die Konstruktionen einen Sonderfall dar. Kein weiteres Kolonialcluster weist eine derart große Anzahl an SN-Token auf. Auf die Einmaligkeit deutschsprachiger Straßenbenennungen der im Zuge des Polenfeldzugs annektierten Stadt wird in Kap. 8.3 diskutiert.

\subsection{Benennungszeiträume}

Die in Dresden verfügten kolonial motivierten SN-Token fallen in die Zeit des faktischen Kolonialismus in der Kaiserzeit und in die Zeit des Nationalsozialismus, während die für Breslau [Wrocław] erhobenen kolonialen Straßenbenen-

chen Gladbach, Münster, Nürnberg, Oberhausen, Saarbrücken, Schweidnitz [Świdnica], Solingen, Stettin [Szczecin], Stuttgart, Waldenburg [Wałbrzych], Wiesbaden, Wuppertal. 
nungen allesamt erst nach der nationalsozialistischen Machtübernahme erfolgten. Auch für das ortsübergreifend zusammengestellte Nameninventar überwiegen administrative Benennungen nach der faktischen Beendigung deutscher Kolonialherrschaft: Weniger als 50 (47) der über 520 SN-Token wurden nachweislich noch während der faktischen Kolonialherrschaft in Übersee verfügt, die sich auf eine überschaubare Anzahl an Großstädten beschränken: Bis auf singuläre Benennungen in den Mittelstädten Cuxhaven und Weimar konnten ein oder mehrere SN-Token nur für Berlin, Bremen, Chemnitz, Dresden, Düsseldorf, Erfurt, Hannover, Köln, Leipzig und Magdeburg mit jeweils über 100.000 Einwohnerinnen und Einwohnern sowie (Berlin-)Dahlem erhoben werden.

Koloniale Straßenbenennung nach der faktischen Abtretung des Kolonialbesitzes ist dagegen als eine usuelle Praxis zu beschreiben: Über $90 \%$ des erstellten Inventars wurden nach 1919 durch die jeweiligen städtischen Administrationen in den öffentlichen Raum des Deutschen Reichs verfügt:

Tab. 6: Verfügungszeiträume des Gesamtinventars kolonial motivierter Straßennamen.

\begin{tabular}{|c|c|c|}
\hline & $\begin{array}{l}\text { Zeitraum der faktischen } \\
\text { Kolonialherrschaft (1884-1919) }\end{array}$ & $\begin{array}{l}\text { Zeitraum nach der faktischen } \\
\text { Kolonialherrschaft (1919-1945) }\end{array}$ \\
\hline Anzahl an SN-Token & 47 & 480 \\
\hline Prozentuale Aufteilung & ca. $9 \%$ & ca. $91 \%$ \\
\hline
\end{tabular}

Die tokenbezogenen Annotationen der 480 nach 1919 verfügten Benennungen nach den jeweiligen gesellschaftspolitischen Einschreibezeiträumen ergibt, dass nur 41 SN-Token des nach 1919 bis 1945 verfügten nachkolonialen Namenbestands in die Zwischenkriegszeit fällt:

Tab. 7: Verfügungszeiträume kolonial motivierter Straßennamen nach der de facto-Herrschaft.

\begin{tabular}{lll}
\hline & Zwischenkriegszeit (1919-1933) & NS-Zeit (1933-1945) \\
\hline Anzahl an SN-Token & 41 & 439 \\
\hline
\end{tabular}

Koloniale Straßenvergaben zwischen 1919 und 1933 bleiben bis auf Altenburg, Frankfurt/Oder und Pirna auf den großstädtischen Raum in Berlin, Bremen, Hannover und Leipzig beschränkt. Erstmalige Benennungspraktiken können darüber hinaus für die Großstädte Gleiwitz [Gliwice], Hamburg, Königsberg i. Pr. bzw. Königsberg (Pr.) [Kaliningrad], München, Nürnberg und Saarbrücken nachgewie- 
sen werden. Koloniale Straßenbenennungen in der Zwischenkriegszeit sind damit wie in der Kaiserzeit als vorrangig großstädtisches Phänomen zu beschreiben.

Nahezu 440 (439) Benennungen des erstellten Inventars sind in die Zeit des Nationalsozialismus zu verorten: Im Hinblick auf die Verteilung des erhobenen Gesamtbestandes bezüglich der Einschreibezeiträume ist die Benennung von Straßen, die in nationalsozialistischer Zeit über ein Jahrzehnt nach dem Abtritt des Kolonialbesitzes Akteure, Orte, Ereignisse u. dgl. der deutschen Kolonialepoche würdigen und/oder ehren, als eine ortsübergreifende Praxis $z u$ beschreiben, die für eine ganze Reihe an Groß- und Mittelstädten erhoben werden konnten: Die zwischen 1933 und 1945 über 430 kolonial motivierten Benennungen sind erstmalig für über 70 Groß- und Mittelstädte festzustellen. Die in nationalsozialistischer Zeit verfügten kolonialen Namenvergabepraktiken sind damit als ortsübergreifend-nationale Phänomene zu beschreiben, die neben weiteren Großstädten auch in einer ganzen Reihe an Mittelstädten Verwendung fanden. Dass der Teilbestand aller in die Mittelstädte verfügten SN-Token mit etwa drei Benennungen pro Stadt weitaus geringer ausfällt als in den Großstädten, wurde bereits in Kap. 6.1 dargelegt.

\subsection{MOD-Types}

\subsubsection{Tokenbezogene Quantitäten}

Die Annotation des Gesamtbestands der über 520 kolonial motivierten Benennungen erfolgte für jedes einzelne SN-Token hinsichtlich der abstrahierten Form als MOD-Type. Das in Kap. 5.2.2.1 erläuterte Verfahren umfasste die Typisierung der linksköpfigen Modifikatoren, damit die Bestandteile anschließend inventarbezogen nach den numerisch dominanten kommemorativen Markern untersucht werden konnten. Der Arbeitsschritt umfasste die Typisierung aller Personennamen durch die entsprechenden Nachnamen, die Vereinheitlichung graphematischer Variationen sowie die Tilgung der unbetonten Schwa-Endung -er bei Städtenamen, die mit silbenstrukturellen Aspekten und der prosodischen Struktur erklärt werden kann. Durch die Typisierungsprozesse konnten für über 520 MOD-Token 96 unterschiedliche ausdrucksseitige MOD-Types zusammengestellt werden, die in der nun folgenden Übersicht in alphabetischer Reihenfolge angeordnet wurden:

Afrikanisch, Anecho, Apia, Archipel, Askari, Bennigsen, Blumenau, Damara, Daressalam, Dattel, (Deutsch-)Ostafrika, (Deutsch-)Südwest(-Afrika), Dominik, Duala, Erckert, Estorff, Finsch, Godeffroy, Gravenreuth, Gröben, Großfriedrichsburg, Groß-Nabas, Haber, Hansemann, Herero, Heydebreck, Iltis, Kakao, Kalahari, Kamerun, Karolinen, Kiautschou, Kibo, 
Kilimandscharo, Kokos, Kolonial, Kolonie, Kongo, Koralle, Kribi, Lans, Lettow-Vorbeck, Leutwein, Liebert, Lome, Lüderitz, Lüderitzland, Maercker, Massai, Meyer-Waldeck, Mohasi, Nachtigal, Nettelbeck, (Neu-)Guinea, Niebuhr, Njassa, Otavi, Palme, Pascha, Peking, Peters, Perlmutter, Riff, Rohlfs, Sambesi, Samoa, Sansibar, Schnee, Senegal, Soden, Solf, Somali, Suaheli, Südost, Südsee, Swakopmund, Tabora, Taifun, Taku(-Fort), Tanga, Tangabucht, Tanganjika, Togo, Transvaal, Tropen, Trotha, Tsingtau, Uganda, Usambara, Vogelsang, Voigts, Waterberg, Weber, Windhuk, Wissmann, Woermann.

Die große Anzahl an MOD-Types lässt zunächst den Eindruck entstehen, dass sich die für kolonial motivierte Fixierungspraktiken verfügten Konstruktionen durch Vielseitigkeit der dabei zum Tragen kommenden kommemorativen Marker auszeichnen. Jenes offenbar hohe Ausmaß an Komplexität wird geschmälert, wenn die jeweiligen MOD-Types hinsichtlich der Anzahl ihrer ortsübergreifend-nationalen Häufigkeitsverteilung an verfügten MOD-Token dargelegt werden: So stellen allein 17 der über 90 mod-Types (Archipel, Dattel, Herero, Kakao, Kalahari, Kokos, Kolonie, Koralle, Njassa, Palme, Perlmutter, Riff, Suaheli, Südost, Taifun, Tanganjika, Tropen) Konstruktionen dar, die in der Verbindung mit usuellen Klassifikatoren ausschließlich für Lodz bzw. Litzmannstadt [€ódź] festzustellen sind (Kap. 8.3). Daneben sind auf weitere 38 singuläre Phänomene hinzuweisen, die als zweigliedrige Konstruktionen mit den usuellen Klassifikatoren -straße, -weg, -platz u. dgl. nur für eine einzige Groß- bzw. Mittelstadt erhoben werden konnten (d. s.: Afrikanisch, Anecho, Apia, Bennigsen, Blumenau, Damara, Estorff, Finsch, Godeffroy, Großfriedrichsburg, Groß-Nabas, Haber, Hansemann, Heydebreck, Karolinen, Kiautschou, Kibo, Kolonial, Kongo, Kribi, Liebert, Lüderitzland, Maercker, Massai, Mohasi, Nettelbeck, Niebuhr, Peking, Sambesi, Schnee, Senegal, Somali, Tabora, Tangabucht, Uganda, Vogelsang, Voigts, Weber). Solche Namen-Einzelfälle spielen zur Beantwortung der mit kolonialen Benennungspraktiken intendierten ortsübergreifend-nationalen Wissenskonzepte eine untergeordnete Rolle. Nur 27 der über 90 MOD-Types sind dagegen drei und über drei Mal als kolonial motivierte Benennungen in der Verbindung usueller Klassifikatoren verfügt worden:

Askari, Daressalam, (Deutsch-)Ostafrika, (Deutsch-)Südwest(-Afrika), Duala, Erckert, Iltis, Kamerun, Lettow-Vorbeck, Leutwein, Lüderitz, Nachtigal, (Neu-)guinea, Otavi, Peters, Rohlfs, Samoa, Sansibar, Swakopmund, Taku(-Fort), Tanga, Togo, Tsingtau, Waterberg, Windhuk, Wissmann, Woermann.

Die tokenbezogene Anzahl des erstellten Gesamtinventars aller kolonial motivierten Namenvergabepraktiken, die jenen 27 MOD-Types zuzuschreiben sind, ist dabei frappierend eindeutig: 444 administrativ verfügte Straßenbenennungen und damit $84 \%$ des erhobenen Inventars sind hinsichtlich der typisierten Modifikatoren auf jene limitierte Auswahl an MOD-Types zuzuschreiben. Durch- 
schnittlich ist jeder dieser 27 MOD-Types nicht durch drei, sondern durch 16 SNToken vertreten. Damit lässt sich ein erster Eindruck davon gewinnen, wie musterhaft sich derartige kolonial motivierte Fixierungspraktiken in den (Alltags-) Raum des Deutschen Reichs im Hinblick auf die bestehenden Konstruktionen gestalten. Auch die modifikatorischen Dubletten der in Dresden und Breslau [Wrocław] verfügten Namen (Lüderitz, Peters, Windhuk, Wissmann) sind innerhalb jener zusammengestellten Liste an 27 MoD-Types vertreten (Kap. 5.6). Die zuvor dargelegten groben prozentualen Angaben sollen das hohe Maß an Systematizität verdeutlichen, das den gewählten Modifikatoren zugesprochen werden muss. Nicht einmal ansatzweise legen derartige Verteilungswerte jedoch die tatsächlichen Distributionen der 444 MOD-Token auf die 27 MOD-Types dar. Das Diagramm zeigt jene tokenbezogenen Häufigkeitsverteilungen der 27 MOD-Types auf, die als zweigliedrige Konstruktionen drei oder mehr als drei Mal bis 1945 in Groß- und Mittelstädte verfügt wurden:

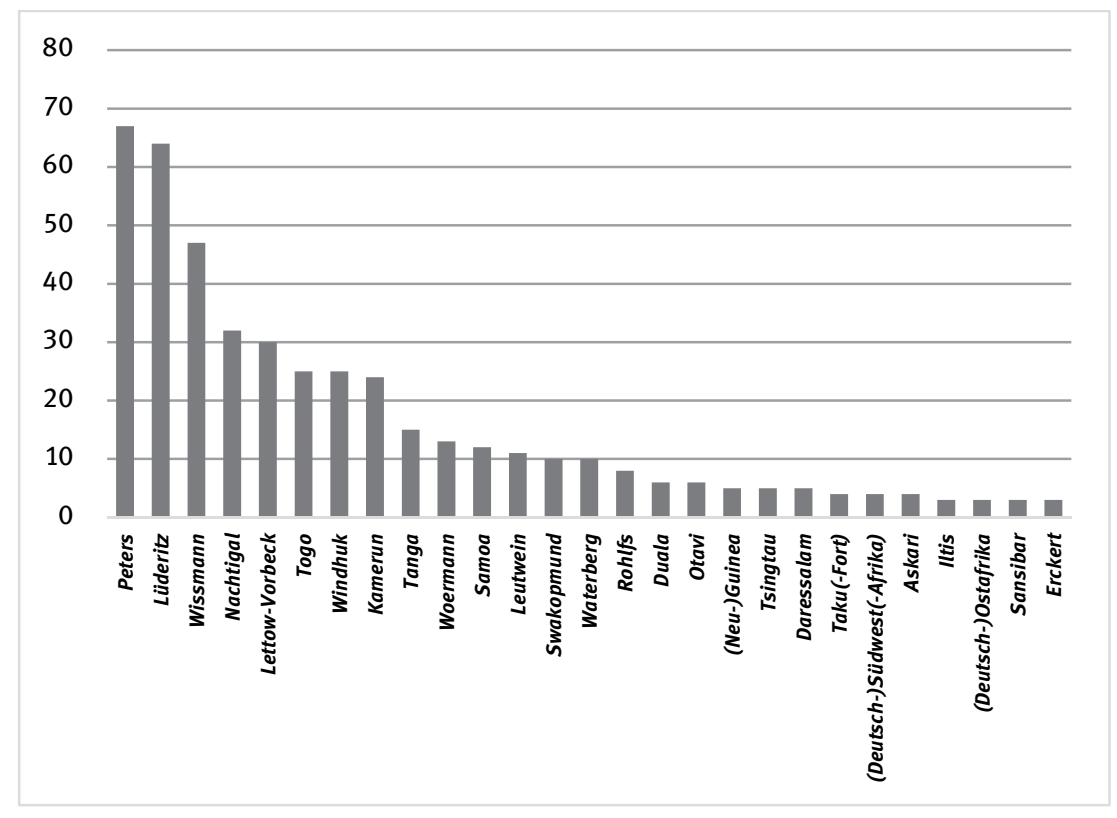

Diagramm 1: 27 ortsübergreifend verfügte MOD-Types (1984-1945) mit den jew. MOD-Token.

Die tokenbezogenen Zahlenwerte sind deshalb so aufschlussreich, weil sich die über 440 Namen hinsichtlich ihrer MOD-Types nicht gleichmäßig verteilen. Stattdessen sind innerhalb dieser 27 Konstruktionstypen wiederum hochfrequente MOD-Types auszumachen: Die acht häufigsten MOD-Types Peters, Lüderitz, Wiss- 
mann, Nachtigal, Lettow-Vorbeck, Togo, Windhuk und Kamerun wurden als zweigliedrige Konstruktionen mit gängigen Klassifikatoren wie -straße, -weg, -platz $\mathrm{u}$. dgl. jeweils über $20 \mathrm{Mal}$ in nachweislich kolonialer Motivik verfügt. Die Produkte kolonialer Namenverfügungspraktiken mit den MOD-Types Peters und Lüderitz sind sogar über 60 Mal festzustellen. Koloniale Straßenbenennungspraktiken im Raum der deutschen Metropole weisen damit hinsichtlich der dabei zum Tragen kommenden kommemorativen Marker einen hohen Grad an Schematismus auf.

\subsubsection{Tokenbezogene Quantitäten in diachroner Perspektive}

Für das erstellte Nameninventar sind in diachroner Perspektive, also in Bezug auf die drei gesellschaftspolitischen Benennungszeiträume, unterschiedliche Distributionen der ortsübergreifend verfügten MOD-Types festzustellen. Der noch während der faktischen Kolonialepoche verfügte Teilbestand von 47 kolonial motivierten SN-Token weist hinsichtlich der betreffenden MOD-Types Distributionen auf, die sich nur partiell mit denen des Gesamtinventars überschneiden: Über die Hälfte jener 47 SN-Token weisen Modifikatoren auf, die nur einzelortsbezogen oder für zwei Orte erhoben werden konnten. Nur fünf MODTypes sind herauszustellen, die innerhalb der de facto-Kolonialherrschaft in der Zeit des Deutschen Kaiserreichs drei oder mehr als drei Mal als zweigliedrige Konstruktionen verfügt wurden:

Tab. 8: Fünf ortsübergreifend verfügte MOD-Types, faktische Kolonialzeit (1884-1919).

\begin{tabular}{llllll}
\hline MOD-Types & Wissmann & Lüderitz & Nachtigal & Rohlfs & Taku(-Fort) \\
\hline Anzahl an SN-Token & 8 & 4 & 3 & 3 & 3 \\
\hline
\end{tabular}

Quantitativ dominant ist der MOD-Type Wissmann, der mit entsprechenden usuellen Klassifikatoren ausschließlich in Großstädten verfügt wurde. Auch für das Gesamtinventar spielt der MOD-Type mit 47 MOD-Token eine übergeordnete Rolle.

Für das Teilinventar der in der Zwischenkriegszeit (1919-1933) verfügten 41 Benennungen konnten sieben MOD-Types erhoben werden, die als tokenbezogene Konstruktionsmuster drei oder mehr als drei Mal verfügt wurden: 
Tab. 9: Sieben ortsübergreifend verfügte MOD-Types, Zwischenkriegszeit (1919-1933).

\begin{tabular}{llllllll}
\hline MoD-Types & Wissmann & Kamerun & Togo & Lüderitz & Nachtigal & Peters & Windhuk \\
\hline $\begin{array}{l}\text { Anzahl an } \\
\text { SN-Token }\end{array}$ & 7 & 4 & 4 & 3 & 3 & 3 & 3 \\
\hline
\end{tabular}

Neben den MOD-Types Wissmann, Lüderitz und Nachtigal sind für den Zeitraum der Weimarer Republik die vier weiteren ortsübergreifend verfügten MOD-Types Kamerun, Togo, Peters und Windhuk herauszustellen, die in der Verbindung usueller Klassifikatoren verfügt wurden. Die jeweiligen Konstruktionen konnten vorrangig für Großstädte, daneben aber auch für einzelne Mittelstädte erhoben werden.

Für den Hauptbestand der 439 in nationalsozialistischer Zeit erfolgten Benennungen entsprechen die ortübergreifend verfügten MOD-Types weitestgehend den Verteilungen des Gesamtinventars.

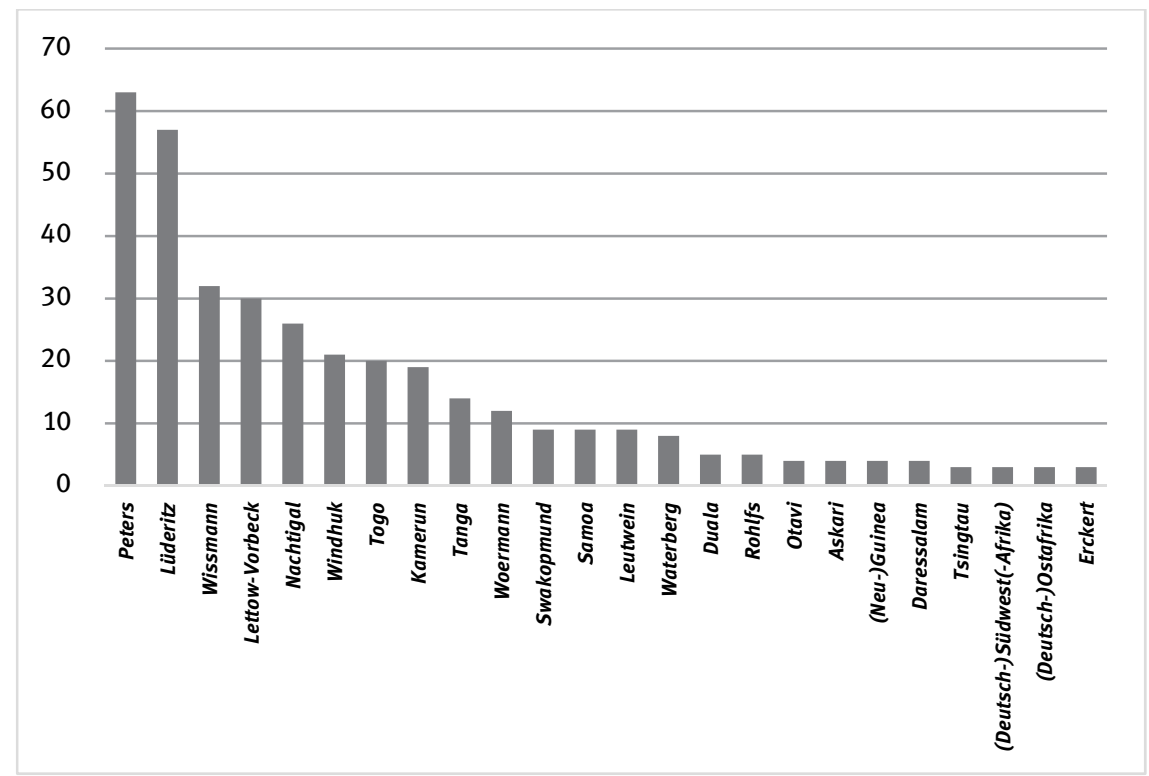

Diagramm 2: Ortsübergreifend verfügte MOD-Types in der NS-Zeit (1933-1945) mit den jew. MOD-Token.

Die beiden Konstruktionsmuster mit den MOD-Types Peters und Lüderitz sind für den jüngsten Benennungszeitraum ebenso als hochfrequent zu verzeichnen. 
Herauszustellen sind die MoD-Types Lettow-Vorbeck, Tanga, Woermann, Swakopmund, Samoa, Leutwein, Waterberg, Duala, Otavi, Askari, (Neu-)Guinea, Daressalam, Tsingtau, (Deutsch-)Südwest(-Afrika), (Deutsch-)Ostafrika und Erckert, die als zweigliedrige Konstruktionen erstmalig innerhalb dieses jüngsten Benennungszeitraums drei und mehr als drei Mal verfügt wurden.

\subsection{Ergebnisse}

Die Annotation des in einem ortsübergreifend-nationalen Zugriff erstellten Inventars führt zu ersten quantitativen Befunden: Die administrative Vergabe kolonial motivierter Straßenbenennungen bis 1945 erweist sich dabei als eine ortsübergreifend-nationale Sprachpraxis im öffentlichen Raum der deutschen Metropole, die für 45 Groß- und 44 Mittelstädte festzustellen ist. Die Verteilung der erhobenen 527 SN-Token hinsichtlich ihrer jeweiligen gesellschaftspolitischen Benennungszeiträume fällt sehr unterschiedlich aus. Für die Zeit der de facto-Kolonialepoche in der Zeit des Deutschen Kaiserreichs und der Zwischenkriegszeit konnten unter 90 SN-Token erhoben werden, die in nachweislich kolonialer Motivik von den städtischen Administrationen verfügt wurden. Die dabei festzustellenden Verteilungen auf die jeweiligen Ortstypen zeigen, dass sich koloniale Namenvergabepraktiken neben wenigen Mittelstädten weitestgehend auf Großstädte verdichten. Im Hinblick auf die Anzahl der bis dato in den städtischen Raum verfügten Kolonialismen steht Berlin als administratives Zentrum der Kolonialbewegung (vgl. Heyden und Zeller 2002: 12) an der Spitze: Für den Zeitraum des faktischen Kolonialbesitzes sind bereits 19 in nachweislich kolonialer Motivik verfügte SN-Token für Berlin festzustellen, die sich bis 1933 auf insgesamt 28 SN-Token ausweiten. Der Vergleich der noch in der faktischen Kolonialzeit maßgeblich in Berlin-Wedding verfügten Konstruktionen mit den für die Zwischenkriegszeit erhobenen ortsübergreifenden Namenverfügungen in weiteren Großstädten und singulären Mittelstädten führt hinsichtlich der MOD-Types zu partiellen Übereinstimmungen. Es kann nur vermutet werden, dass die Konstruktionsmuster des jüngeren Benennungszeitraums zum Teil auf Nachahmung (vgl. Tarde 2009) der in der ehemaligen Kolonialreichshauptstadt Berlin verfügten älteren Kolonialismen beruhen. Über 430 SN-Token und damit etwa $83 \%$ des erhobenen (Gesamt-)Inventars wurde dagegen erst nach 1933 in eine beträchtliche Anzahl an Groß- und Mittelstädten verfügt. Der Großteil jenes in nationalsozialistischer Zeit verfügten Namenbestandes referiert damit zum Benennungszeitpunkt auf vergangene koloniale Machtkonstellationen des Deutschen Kaiserreichs, die bereits durch die im Friedensvertrag von Versailles im Juni 1919 festgelegten Bestimmungen beendet wurden. Die für zehn Orte in 
den annektierten Gebieten erhobenen kolonialzeitbezogenen Namen fallen hinsichtlich ihrer Anzahl an Benennungen sehr unterschiedlich aus: Neben einzelnen Kolonialismen sind für einige Städte eine Vielzahl an kolonialzeitbezogenen SN-Token zu konstatieren. Das in Lodz bzw. Litzmannstadt [Łódź] erhobene Kolonialcluster mit nahezu 30 SN-Token ist nicht nur aufgrund der Höchstzahl an kolonialen Straßennamen markiert, sondern auch deshalb, weil eine ganze Reihe an Konstruktionen festzustellen ist, die aus gesamtinventarbezogener Perspektive einzig und allein für die nach dem Überfall auf Polen annektierte Stadt festzustellen sind.

Für das erstellte (Gesamt-)Inventar sind 27 MOD-Types herauszustellen, die über drei Mal in der Verbindung usueller Klassifikatoren verfügt und über 440 Konstruktionen zuzurechnen sind. Jenes hohe Maß an Systematizität ist für sich schon ein nennenswertes Ergebnis: Zur Beantwortung der übergeordneten Leitfrage wurde eine Datengrundlage geschaffen, die es erlaubt, jene vorerst quantitativen Betrachtungen mit weiteren Analysen hinsichtlich der damit versprachlichten kolonisatorischen Gewissheiten zu untersuchen. Im folgenden Kapitel wird dargelegt, welche lexikologisch-onymischen Klassen maßgeblich in die über 520 Modifikator-Konstruktionen eingehen, um sodann übergeordnete Aussagen über die damit etablierten kolonialtoponomastischen Diskursfunktionen treffen zu können. 